\title{
The Effectiveness of Mobilisation with Movement for Chronic Medial Ankle Pain: A Case Study
}

\author{
A case report submitted to the Orthopaedic Manipulative Therapists' Group of the South African Society \\ of Physiotherapy, in partial fulfilment of the requirements for the Continuing Education Course \\ in Orthopaedic Manipulative Therapy (OMT 1). Bloemfontein, October 2006.
}

\begin{abstract}
Introduction and Purpose: It has been shown that approximately thirty percent of those sustaining an ankle sprain are likely to develop chronic functional limitations. Mulligan has developed mobilisation with movement (MWM) for treatment of joint dysfunction and suggests that it is a positional fault of the joint that causes pain and movement restriction.

Method: This single case reports on the effects of a MWM technique on chronic medial ankle pain. The patient was a 25-year-old female runner who had experienced

\section{Penso M, BSc (Physio) UCT,} OMT 1 Bloem ${ }^{1}$

${ }^{1}$ University of Cape Town. pain since an initial ankle sprain at 8 years of age. The main findings of the subjective and physical examinations were decrease in active and passive dorsiflexion and eversion range of motion (ROM), shortening of gastrocnemius and soleus muscles and the functional limitation of pain when running. The patient was treated twice with the MWM technique.
\end{abstract}

Results: Outcomes of immediate reduction in pain, restoration of full dorsiflexion and eversion ROM, increase in calf muscle length and pain free running were observed. This was maintained over a four-month follow up period.

Conclusion: This case concurs with previous studies detailing increases in range of motion and restoration of pain free movement as well as adding new support for the resolution of chronic pain with MWM.

\section{KEY WORDS: MOBILISATION WITH MOVEMENT, MULLIGAN, CHRONIC ANKLE PAIN.}

\section{INTRODUCTION AND LITERATURE REVIEW}

The subject of this case study presented with chronic medial ankle pain with a history of a serious ankle sprain as a child. Ankle sprains, especially of the lateral ankle ligaments, are extremely common injuries in both the athletic and the general population (Brukner and Khan 2001, Kirchner et al 2005). Approximately thirty percent of those sustaining an ankle sprain are likely to develop chronic functional limitations post injury regardless of whether treatment was previously received (Kavanagh 1999, Kirchner et al 2005). Pain and/or instability especially with more stressful activities, such as running, are frequent complaints.

Management of chronic conditions often poses a challenge to the physiotherapist. Chronic ankle pain can be attributed to inadequate rehabilitation or misdiagnosed underlying injury. Accurate history taking and examination of the ankle will guide in the appropriate management (Brukner and Khan 2001, Kirchner et al 2005). Loss of proprioception, dorsiflexion range of motion (ROM) and posterior talar glide are common findings in chronic ankle sprain. The decreased dorsiflexion ROM actually predisposing to recurring ankle injuries (Vicenzino et al 2006).

The literature describes conventional treatment of chronic ankle sprains to include physical therapy for active and passive joint mobilisation (Brantingham et al 1993, Maitland 1977), muscle strengthening and proprioceptive training (Brukner and Khan 2001), orthotics to correct pronation (Brantingham et al 1993), and surgery (Brukner and Khan 2001, Kirchner et al 2005).

Mulligan (1993) developed "mobilisation with movement" (MWM) for treatment of joint dysfunction and suggests that it is a positional fault of the joint that causes pain and movement restriction. This hypothesis has been supported by research in three different studies (Hetherington 1996, Kavanagh 1999, Merlin et al 2005). MWM describes a manually sustained accessory glide parallel or perpendicular to the treatment plane applied while the patient performs the painful physiological movement. MWM must render the symptomatic movement pain free and must increase the restricted ROM. The correction can then be maintained with taping and rehabilitation.

Articles published have described the favourable effects of MWM on acute and subacute lateral ankle sprains (Collins et al 2004, O'Brien and Vicenzino 1998), the correction of a positional fault in the distal tibiofibular joint post ankle sprain (Hetherington 1996, Hubbard et al 2006, Kavanagh 1999, Merlin et al 2005), the effect of MWM on dorsiflexion range (Vicenzino et al 2001), and dorsiflexion and posterior talar glide (Vicenzino et al 2006). The effect of MWM on the chronic condition of tennis elbow has also been researched (Vicenzino et al 2001, Vicenzino and Wright 1995). However, there has not, to the knowledge of the author, been a
CORRESPONDENCE TO:
Marian Penso
5 Glen Crescent,
Oranjezicht, 8001
Email: marianpenso@gmail.com
Cell: 083 488-3153
Tel Home: (021) 424-1536 
study on the effect of MWM on chronic medial ankle pain.

The aim of this study was to assess the effect of MWM used for management of a runner complaining of chronic medial ankle pain. A single case study design was used.

\section{CASE REPORT}

\section{Case History}

A 25-year-old female runner presented at the physiotherapy rooms complaining of chronic medial left ankle pain felt on her 7-10 km twice-weekly run. She complained of localised sharp 8/10 pain with the first five minutes of running, when running uphill and on the right hand side of the road. Stretching her left calf muscle also aggravated her pain. The pain was eased by walking for the first five minutes of the run (warm up) and limiting her dorsiflexion range.

The patient had a chronic history of ankle sprains having first sprained her ankle "badly" (plantarflexion inversion injury) at the age of 8 years, when she was unable to walk on the left ankle for 3 weeks or run for a month. No treatment was received. She had since sprained her left ankle numerous times, yet never to the same extent and not within the past two years. She had, thus, suffered from the current complaint intermittently for 17 years but, until now, never had it investigated. The only past medical history of note was bilateral shin splints four years previously, the symptoms of which had been completely relieved by wearing anti-pronation running shoes.

The patient was not suffering from any other medical complaints, was not experiencing any neurological symptoms nor was she taking any medication. No x-rays or other investigations had been performed.

\section{Subjective Examination}

On subjective examination the patient presented with two main "yellow flags". The first being that she was irritated that she had to warm up before her run, which limited her from running with a club (for the fear of appearing "weak"). The second was that she had taken a very long time to seek treatment and was almost in denial of her pain.

The three activities that the patient identified on the Patient-Specific Functional Scale (1998 version) were pain with running, pain with hiking uphill and ineffective stretching of her left calf muscle.

The patient's main functional goal was to be able to run 7-10 km twice a week pain free and without first having to warm up for 5 minutes.

\section{Physical Examination}

Observed abnormalities in the patient's standing posture included a decrease in left knee extension, slight external rotation of the left tibia, increased weight bearing on the right foot, bilateral supination of feet and bilateral high arches.

A visual analysis of the patient's barefoot running gait showed the following deviations: bilateral pronation, decreased strike of her left heel at heel strike (toe running), eversion of left ankle with swing through, uneven cadence and heavy landing of her right foot in midstance.

The squat revealed an obvious decrease in dorsiflexion range and the patient reported that the left ankle felt "stuck".

Active and passive movements of the left ankle were limited, the most marked being dorsiflexion and eversion. Refer to table 1 .

Calf muscle length was limited on the left for both gastrocnemius and soleus muscles. Refer to table 2. The length was measured with a standard ruler over a $10 \mathrm{~cm}$ step as the distance from the floor to the lowest part of the patient's heel. In prone the difference in the patient's heel height between left and right sides was also measured. The measurements were taken three times and the average recorded.

Tests as described in Petty and Moore (1998) were used. The anterior drawer sign and talar tilt were negative indicating uninterrupted ankle ligaments. The patient's lower limb was neurologically intact and neurodynamic testing of straight leg raise revealed no neurodynamic mobility problems with sensitisation. One leg standing test for proprioception showed good balance and proprioception on both legs.

On palpation the left ankle was slightly cold and clammy, but no oedema or swelling was noted. Tenderness of 2/10 was elicited over the anterior medial area (over the talus) of the left ankle with deep pressure. This area also felt thickened compared to the right side and the talus felt medially displaced. Tightness of the proximal gastrocnemius and soleus was felt compared to the right side. Palpations of the Achilles tendon, medial and lateral ankle ligaments, the extensor retinaculum, and tibialus anterior tendon were all non-tender.

Posteroanterior (PA) accessory movement of the talocrural joint elicited the patient's sharp $8 / 10$ pain at half ROM with a blocked end-feel, the other movements were full range and pain free. Accessory examination of the tarsal, metatarsal, phalangeal, subtalar, and proximal and distal tibiofibular joints were asymptomatic.

Clearing tests as described by Petty and Moore (1998) were asymptomatic for the patient's lumbar spine, sacroiliac joint, hip and knee.

\section{Planning of intervention}

On completion of the physical examination the patient's pain was severe when provoked yet non-irritable as it eased within seconds. The pain was more mechanical in nature due to reproduction through movement. The three main findings of the subjective and physical examinations were decrease in active

Table 1: Movement tests

\begin{tabular}{|c|c|c|c|c|c|c|}
\hline & $\begin{array}{l}\text { Active } \\
\text { ROM }\end{array}$ & & & $\begin{array}{l}\text { Passive } \\
\text { ROM }\end{array}$ & & \\
\hline & Left & Response & Right & Left & Response & Right \\
\hline Dorsiflexion & $21^{\circ}$ & $8 / 10$ sharp pain ( $\mathrm{A} 1$ and $\mathrm{A} 2$ ) & $35^{\circ}$ & $21^{\circ}$ & $\begin{array}{l}\text { 8/10 sharp pain ( } \mathrm{A} 1 \text { and } \\
\mathrm{A} 2 \text { ) blocked end-feel }\end{array}$ & $38^{\circ}$ \\
\hline Plantarflexion & $40^{\circ}$ & Controlled movement, no pain & $45^{\circ}$ & $45^{\circ}$ & Normal end-feel. No pain & $45^{\circ}$ \\
\hline Inversion & $21^{\circ}$ & Controlled movement, no pain & $30^{\circ}$ & $28^{\circ}$ & Normal end-feel. No pain & $33^{\circ}$ \\
\hline Eversion & $16^{\circ}$ & 2/10 pulling pain (A2) & $32^{\circ}$ & $17^{0}$ & $\begin{array}{l}\text { 5/10 pain (A2). } \\
\text { Blocked end-feel }\end{array}$ & $35^{\circ}$ \\
\hline
\end{tabular}

Key: A1 anterior medial area of left ankle, A2 posterior medial area of left ankle 
and passive dorsiflexion and eversion, accessory PA of talocrural joint, and measured calf muscle shortening. No concerns for misdiagnosed underlying conditions were found.

It was hypothesised that displacement or a "positional fault" of the talocrural joint had occurred with the initial ankle sprain at age 8 . This was now blocking full ankle ROM and causing pain with running. The hypothesised pathobiological pain mechanisms involved were primarily of nociceptive and motor origin (altered running gait, shortening of calf muscle) with contribution from cognitive and affective processing mechanisms (pushing to available range, playing down condition) and autonomic outputs (cold and clammy foot).

The aim of the management was to reduce the patient's pain and increase her range of dorsiflexion by correcting the positional fault of the talocrural joint. Calf muscle shortening would also have to be addressed. This would allow for achievement of the patient's functional goal. A technique that did not provoke her severe pain, yet went into resistance (due to the chronic nature of the condition), would be ideal for treatment. Rehabilitation would include promotion of good running style with heel strike, an effective calf stretching program and educating the patient on the importance of seeking early treatment of an injury. The patient would be referred for x-rays if her pain persisted (rule out fracture or other complication).

\section{Management}

The patient was treated twice over a two-week period and then seen one month and four months later for follow up. The patient continued to run 7 to $10 \mathrm{~km}$ twice weekly during the intervention.

The initial technique tried was the specific technique described by Mulligan (2004) to increase dorsiflexion ROM in weight bearing. However, this did not relieve the patient's pain even though, as suggested by Mulligan, subtle changes in direction of the glide were tried. So in keeping with Mulligan's treatment rule a glide in the opposite direction was employed.

The treatment technique used was an anterior-posterior glide applied to the distal tibia with stabilisation of the posterior foot while the patient performed active dorsiflexion in weight bearing on a step, executed into resistance, but without pain. Weight bearing

Table 2: Calf muscle length (distance from heel to ground off $10 \mathrm{~cm}$ step)

\begin{tabular}{|l|l|l|}
\hline & Left & Right \\
\hline Gastrocnemius & $3.25 \mathrm{~cm}$ & $2.75 \mathrm{~cm}$ \\
\hline Soleus & $4.25 \mathrm{~cm}$ & $3.5 \mathrm{~cm}$ \\
\hline
\end{tabular}

was chosen as Mulligan (2004) suggests that the movement gained in weight bearing is maintained. The technique was performed ten times and then repeated, thereafter the outcome measures assessed.

After the second treatment session the patient was instructed on maintaining her new calf muscle length with stretching. The patient was to place her hands on a wall with her left leg behind the right in a manner as to feel a stretch in her calf and hold the stretch for three times thirty seconds. This was to be performed with her knee straight and then bent and done once a day as well as post running. The patient adhered to these stretches. The patient was also encouraged to run with proper heel strike. It was unnecessary to refer for $\mathrm{x}$-rays.

\section{OUTCOMES}

Refer to tables 3-5 for outcomes in ROM, muscle length and function.

\section{DISCUSSION}

This study has shown the beneficial effects of applying a MWM technique to manage chronic medial ankle pain. This technique, based on the principles pioneered by Mulligan (1993), produced immediate relief of pain with accessory PA movement and weight bearing dorsiflexion as well as an increase in ankle dorsiflexion and eversion ROM. An unexpected improvement in both gastrocnemius and soleus muscle length

\section{Table 3: Pain-free ROM}

\begin{tabular}{|l|l|l|l|l|}
\hline ROM (pain-free) & First treatment & Second treatment & One month & Four months \\
\hline Dorsiflexion & $29^{\circ}$ active and passive & $\begin{array}{l}36^{\circ} \text { active and } \\
37^{\circ} \text { passive }\end{array}$ & $\begin{array}{l}35^{\circ} \text { active and } \\
36^{\circ} \text { passive }\end{array}$ & $\begin{array}{l}35^{\circ} \text { active and } \\
36^{\circ} \text { passive }\end{array}$ \\
\hline Plantarflexion & $45^{\circ}$ active and passive & $45^{\circ}$ active and passive & $45^{\circ}$ active and passive & $45^{\circ}$ active and passive \\
\hline Inversion & $30^{\circ}$ active and passive & $33^{\circ}$ active and passive & $33^{\circ}$ active and passive & $33^{\circ}$ active and passive \\
\hline Eversion & $32^{\circ}$ active and passive & $36^{\circ}$ active and passive & $35^{\circ}$ active and passive & $35^{\circ}$ active and passive \\
\hline
\end{tabular}

Table 4: Muscle length

\begin{tabular}{|l|l|l|l|l|}
\hline Muscle Length & First treatment & Second treatment & One month & Four months \\
\hline Gastrocnemius & $2.75 \mathrm{~cm}$ & $2.5 \mathrm{~cm}$ & $2.5 \mathrm{~cm}$ & $2.5 \mathrm{~cm}$ \\
\hline Soleus & $3.75 \mathrm{~cm}$ & $3.5 \mathrm{~cm}$ & $3.5 \mathrm{~cm}$ & $3.5 \mathrm{~cm}$ \\
\hline Difference in heel height & $2 \mathrm{~cm}$ & $0.5 \mathrm{~cm}$ & $0 \mathrm{~cm}$ & $0 \mathrm{~cm}$ \\
\hline
\end{tabular}

Table 5: Functional improvements as adapted from the Patient-Specific Functional Scale

\begin{tabular}{|l|l|l|l|l|}
\hline Function & First treatment & Second treatment & One month & Four months \\
\hline Running & 2 minute warm up required & No need for initial warm up & $\begin{array}{l}\text { Able to run on right side of } \\
\text { road and uphill pain-free }\end{array}$ & $\begin{array}{l}\text { Pain-free running main- } \\
\text { tained, able to run with club }\end{array}$ \\
\hline Hiking & $4 / 10$ pain on uphill & $2 / 10$ pain on uphill & $\begin{array}{l}\text { No pain on uphill, } \\
\text { steadier on downhill }\end{array}$ & Able to hike in a group \\
\hline Effective calf stretch & $1 / 10$ pain with stretching & Able to feel stretch in calf & Efective stretching of calf & $\begin{array}{l}\text { Pain-free effective } \\
\text { stretching maintained }\end{array}$ \\
\hline
\end{tabular}


was also measured. The improvement in these impairments restored the patient's ability to run pain-free. Resolution of this disability enabled the patient to participate in club running and hiking in a group.

This case is consistent with current research showing that MWM to the ankle provides immediate pain relief and increases dorsiflexion ROM (O'Brien and Vicenzino 1998, Vicenzino et al 2001) but to the knowledge of the author, is the first study to note the restoration of eversion ROM and calf muscle length. It is also, to the knowledge of the author, the first study to verify Mulligan's claim that MWM can resolve a chronic condition.

As the impairments improved so functional activity improved. Twenty degrees of ankle dorsiflexion is needed in normal running gait (Inman as cited in Klingman 1999) thus resulting in altered running style and pain. A restoration of this range reduced pain and restored heel strike.

The patient also reported no longer having pain when running on the right hand side of the road. On the right side of the road, the camber slopes down to the right, requiring eversion of the left ankle. Due to the patient's loss of eversion ROM this activity caused pain. When restored the pain dissipated. The patient also reported improvement in perceived unsteadiness with downhill hiking. As the patient's proprioception was intact this could be attributed to the neuro-muscular facilitation effect of MWM proposed by Exelby (1996) and Wilson (1995).

Both gastrocnemius and soleus muscle length improved. Exelby (1996) states that joint mobilisations performed into resistance but without excessive pain have an effect on muscle activity and length and suggests that through MWM, a passive pain free end-range corrective joint glide with an active movement, muscle length can be influenced. Mulligan (2004) suggests that there is no such thing as adaptive muscle shortening in chronic conditions. In this study the calf could never be effectively stretched due to the patient's decreased dorsiflexion ROM. Thus as the dorsiflexion ROM improved so the calf muscle "lengthened". This then supports Mulligan's theory.

Mulligan (1993) attributed the rapid restoration of pain-free function with
MWM to correction of a bony positional fault (occurring in the joint post injury). In this study the patient reported that the joint felt "stuck" with forced dorsiflexion and examination revealed altered talar position, a blocked end feel with a specific accessory movement as well as only one direction in glide that relieved the patient's pain. These findings were indicative of a positional fault. In this study two treatment sessions alleviated and maintained the patient's complaint making MWM very time and cost effective. It would be interesting to note if the correction in positional fault will prevent the patient from re-spraining her ankle in future.

The first MWM tried in the intervention was a posterior-anterior glide on the distal tibia and fibula as described by Mulligan, yet this was ineffective. The action of this glide would restore posterior talar glide, which is commonly lost in chronic ankle sprain (Vicenzino et al 2006). However, in this case the patient presented with loss of anterior talar glide (PA accessory movement of the talocrural joint elicited the patient's $8 / 10$ sharp pain) and thus the glide that restored pain-free range was an anteriorposterior glide to the distal tibia and fibular.

A limitation of this study was the accurate measurement of ROM and muscle length. It is suggested that future studies make use of the dorsiflexion measure (weight bearing with knee against a wall) described by Vicenzino et al (2001).

\section{CONCLUSION}

In this study a patient that had suffered chronic medial ankle pain while running over a period of 17 years was now able to continue with her activity without pain after simple mobilisation with movement intervention. The MWM technique was effective in abolishing pain and increasing ankle ROM. A surprising improvement in calf muscle length was also observed. Although the single case study design limits generalisation of its findings, it is suggested that a patient presenting with chronic medial ankle pain be assessed for possible positional fault of the ankle and, if present, managed with MWM. Research into the effects of MWM on chronic conditions and the mechanism by which this technique works requires further investigation.

\section{REFERENCES}

Brantingham J, Snyder R, Wong J, Brantingham C, Haggart B 1993 Chronic ankle pain: unresolved ankle sprain part I and II. http://www.chiroweb. com/archives/11/02/16.html (2006/10/05)

Brukner P, Khan K 2001 Clinical Sports Medicine, 2nd edn. McGraw-Hill, Australia. pp 51-66, 553-583 Collins NB, Teys P, Vicenzino B 2004 The initial effects of a Mulligan's mobilization with movement technique on dorsiflexion and pain in subacute ankle sprains. Manual Therapy 9(2):77-82 Exelby L 1996 Peripheral mobilisations with movement. Manual Therapy 1:118-126

Hetherington BH 1996 Lateral ligament strains of the ankle, do they exist? Manual Therapy 1(5):274-275

Hubbard T, Hertel J, Sherbondy P (2006) Fibular position in individuals with self-reported chronic ankle instability. Journal of Orthopaedic and Sports Physical Therapy 36(1):3-9

Kavanagh J 1999 Is there a positional fault at the inferior tibiofibular joint in patients with acute or chronic ankle sprains compared to normals? Manual Therapy 4(1):19-24

Kirchner JS, Musgrave AL, Musgrave DS 2005 Chronic pain continues after ankle sprain resolution. http://biomech.com/printable/index.jhtml?articleID= $173500601(2006 / 10 / 05)$

Klingman RE 1999 The pseudoradicular syndrome: a case report implicating double crush mechanisms in peripheral nerve tissue of the lower extremity. The Journal of Manual and Manipulative Therapy 7(2):81-91

Maitland GD 1977 Peripheral Manipulation, 2nd edn. Butterworths, Chapel River Press, Great Britain. pp 272-286

Merlin D, McEwan I, Thom J 2005 Mulligan's mobilisation with movement technique for lateral ankle pain and the use of magnetic resonance imaging to evaluate the "positional fault" hypothesis. XIV International Congress on Sports Rehabilitation and Traumatology: The accelerated rehabilitation of the injured athlete. www.isokinetic. com/pdf_attivita/ 2005/2005-053.pdf (2006/07/21) Mulligan BR 2004 Manual Therapy "NAGS", "SNAGS", "MWM" etc. 5th edn. Plane View Services Ltd, Wellington, New Zealand

Mulligan BR 1993 Mobilisations with movement (MWM'S). the Journal of Manual and Manipulative Therapy 1(4):154-156

O'Brien T, Vicenzino B 1998 A study of the effects of Mulligan's mobilization with movement treatment of lateral ankle pain using a case study design. Manual Therapy 3(2):78-84

Petty N, Moore A 1998 Neuromusculoskeletal Examination and Assessment: A handbook for therapists. Churchill Livingstone, The Bath Press, Bath. pp 49, 313-334

Vicenzino B, Branjerdporn M, Teys P, Jordan K 2006 Initial changes in posterior talar glide and dorsiflexion of the ankle after mobilization with movement in individuals with recurrent ankle sprain. Journal of Orthopaedic and Sports Physical Therapy 36(7):464-471

Vicenzino B, Paungmali A, Buratowski S, Wright A 2001 Specific manipulative therapy treatment for chronic lateral epicondylalgia produces uniquely characteristic hypoalgesia. Manual Therapy 6(4):205-212

Vicenzino B, Prangley I, Martin D 2001 The initial effect of two Mulligan mobilisation with movement treatment techniques on ankle dorsiflexion. www.ausport.gov.au/fulltext/2001/acsms/paper/ VICE2.pdf (2006/07/21)

Vicenzino B, Wright A 1995 Effects of a novel manipulative physiotherapy technique on tennis elbow: a single case study. Manual Therapy 1:30-35

Wilson E 1995 Mobilisation with movement and adverse neural tension: an exploration of possible links. Manipulative Physiotherapist 27(1):40-44 